The strength of potentially relevant associations, and therefore their informativeness, is diminished by misclassification and a specific (ie, minimise false positives) is preferred to a sensitive (ie, minimise false negatives) case definition. Similarly, where possible, objective evidence is desirable of potentially relevant factors such as the date of onset of symptoms, job title, tasks and site of work and dates of introduction of new or changed processes. The case definitions used in the study reported by Robertson and colleagues are clearly defined but, inevitably, are more specific for alveolitis than for asthma. The authors conclude that the most probable cause of the outbreak was contamination (probably microbial) of aerosolised MWF widely used in the factory. Consistent with this conclusion, those who worked in machining and used MWF from the largest common sump had a 3.5-fold greater risk of having disease $(27.3 \%)$ than those working in other parts of the factory $(7.9 \%)$, although this relationship was weaker for allergic alveolitis than for occupational asthma. The authors were reliant on questionnaire responses (and therefore personal recollection) for the date of onset of breathlessness, the largest number reporting this to have been March 2003 which preceded by 1 month a steep rise in tramp oil in MWF in the large communal sump as shown in the factory records.

While, on the basis of their findings, contamination of MWF seems the most probable explanation, as in the majority of US studies the authors were not able to identify a plausible specific responsible agent. Levels of exposure to airborne MWF in the factory were generally below the current Health and Safety Executive guidance value. However, a number of micro-organisms were identified in MWF taken from the largest common sump, in particular Acinetobacter spp and Ochrabactrum anthropi. 59\% of cases of EAA had precipitating antibodies to one of these species or to used sump oil compared with $10 \%$ of cases of occupational asthma and 5\% of asymptomatic (control) workers. The absence of an identified specific cause limits the potential for specific control measures, but is sufficient to warrant a focus on minimising microbial contamination of MWFs where these are used.

It remains unclear why this outbreak occurred. What led to a change, presumably in the microbial flora in MWF, sufficient to cause the outbreak? If future outbreaks occur, it would be interesting to make a comparison-not just between cases and non-cases in the factory with disease-but also between factories with disease ("case" factories) and factories also using MWFs but without cases of disease ("control" factories). Such comparisons could illuminate an underlying cause.

History tells us that precise identification of the specific cause of the disease is not essential to initiate effective control measures. Snow's recognition that water contaminated with sewage in the Thames was the cause of the outbreaks of cholera he investigated led to the initiation of effective measures to prevent the transmission of the disease many years before the identification of a bacterium as its cause.

Thorax 2007;62:928-929.

doi: $10.1136 /$ thx. 2007.082362

Correspondence to: Professor A J Newman Taylor, National Heart and Lung Institute, Imperial College, Guy Scadding Building, Dovehouse Street, London SW3 6LY, UK; a.newmant@ imperial.ac.uk

Competing interests: None.

\section{REFERENCES}

1 Snow J. On the mode of communication of cholera, 2nd edn. London: Churchill, 1855.

2 Kreiss K, Gomaa A, Kullman G, et al. Clinical bronchiolitis obliterans in workers at a microwave popcorn plant. N Engl J Med 2002;347:330-8.

3 Moya C, Anto JM, Newman Taylor AJ, and the Collaborative Group for the Toxicity in Textile Aerographic Factories. Outbreak of organising pneumonia in textile printing sprayers. Lancet 1994;344:498-502.

4 Kern DG, Crausman RS, Durand KT, et al. Flock worker's lung: chronic interstitial lung disease in the nylon flocking industry. Ann Intern Med 1998; 129:261-72.

5 Cullinan P, Harris JM, Newman Taylor AJ, et al. An outbreak of asthma in a modern detergent factory. Lancet 2000;356:1899-900.

6 Bernstein DI, Lummus Zl, Santilli G, et al. Machine operator's lung: a hypersensitivity pneumonitis disorder associated with exposure to metal working fluid aerosols. Chest 1995;108:636-41.

7 Robertson W, Robertson AS, Burge CBSG, et al. Clinical investigation of an outbreak of alveolitis and asthma in a car engine manufacturing plant. Thorax 2007;62:981-90.

8 Fox J, Anderson H, Moen T, et al. Metal working fluid-associated hypersensitivity pneumonitis: an outbreak, investigation and case-control study. Am J Ind Med 1999;35:58-67.

9 Hodgson MJ, Bracker A, Yang C, et al. Hypersensitivity pneumonitis in a metal-working environment. Am J Ind Med 2001;39:616-28.

\title{
Risk and benefit: the eternal Yin and Yang of thoracic surgery
}

\author{
Alex G Little
}

Quality of life, as well as its length, is an essential consideration in developing and recommending therapeutic strategies

W ith the occasional exception, non-small cell lung cancer (NSCLC) is not curable without some form of surgical resection. The good news-ie, the benefit-is that when appropriate oncological standards are followed, cure can be obtained in a substantial majority of operated patients. However, this benefit is not gained without exposing the patient to risk. This risk/benefit relationship is the yin and yang-the two opposing considerations-of thoracic surgery. The proximate risks associated with lung resection are morbidity and perioperative mortality. The challenge, both for a given individual and for large populations, is to maximise the likelihood of benefit while minimising the risk. The paper by Strand et al ${ }^{1}$ in this issue of Thorax (see page 991) addresses the issue of operative risk with regard to 30-day postoperative mortality. This paper makes a major contribution to this issue by clearly defining specific risk factors and even developing a helpful model to estimate the risk of postoperative death.

This Norwegian study reviewed a total of 4395 patients who underwent some form of lung resection for NSCLC between the years 1993 and 2005. Considerable data were able to be reviewed because of the Norwegian law that newly diagnosed cases of cancer have to be reported to the Cancer Registry of Norway. Mining this database for patients with NSCLC revealed the following conclusions. While the overall 30-day postoperative mortality rate was $4.4 \%$, the mortality decreased during the study period, demonstrating a continually diminishing risk of death. This rate seems appropriate as it is nearly identical to the 
$4.1 \%$ mortality found in a similar analysis of patients in 2001 in the USA. ${ }^{2}$

Statistically significant risk factors for patients undergoing lung resection for cancer included male gender, increasing age, operations on the right lung and the need for more extensive operations such as pneumonectomy or bilobectomy. In addition, patients with a higher Charlson co-morbidity index had a higher risk of postoperative mortality than patients with a lower index. These findings were used to generate a prediction model which can be used to define the risk of 30-day postoperative mortality for any specific patient. Knowing these risk factors and having the risk formula will be useful for thoracic surgeons and provide the opportunity for an evidence-based selection of patients for surgery. However, an important caveat which the authors make is that "... surgical treatment should not be withheld because of co-morbid conditions ... alone". In other words, there is still need for art as well as science.

Some potential risk factors were not conclusively defined. First, the relationship between surgeon specialty and outcomes could not be identified any more specifically than to say that some were general surgeons and some were cardiothoracic surgeons. This relationship, of course, would be interesting to know as previously reported studies have found better outcomes for dedicated thoracic surgeons. ${ }^{3}{ }^{4}$ Two other risk and outcome considerations which have been the target of inconclusive international debate could not be definitively resolved by this study ${ }^{5}$-namely, the type of hospital (general vs university) and the question of hospital volume. The $\mathrm{p}$ values for both these analyses approached but did not reach statistical significance. The question therefore remains of the possibility of a $\beta$-type error and that, with a larger dataset, both of these differences might in fact reach statistical significance. The issue is also clouded by the fact that most of the Norwegian highvolume hospitals were also university hospitals, confounding the issue even further. The questions of the roles of these possible determinants of outcome, surgeon specialisation, hospital type and hospital volume remain unsettled.

This influential study emphatically highlights the value and importance of tracking patient outcomes in a prospective database. This report and its observations would not have been possible without the government-required Cancer Registry of Norway. In the USA the two equivalents are the Society of Thoracic Surgeons database and the American College of Surgeons' national cancer database. Participation in these databases allows individual surgeons, surgical groups and hospitals to compare their outcomes with national outcomes which, in turn, allow them to identify areas where performance and quality can and should be improved. Further, these large databases allow determination of risk adjusted mortality and morbidity rates, which are the only legitimate and reasonable way to compare, stratify and determine appropriate outcomes.

One factor not considered by this report is the distinction between the length of life and its quality. For any comprehensive consideration of the risk-benefit of lung resection for patients with lung cancer, follow-up beyond 30 days and, in fact, for a lifetime is therefore essential. If the patient survives but experiences severe post-thoracotomy pain, becomes a respiratory cripple, or is unable to resume the desired lifestyle, the "benefit" of a curative operation is compromised. The complete picture must be kept in mind. Quality of life, as well as its length, is an essential consideration in developing and recommending therapeutic strategies. ${ }^{6}$

Thorax 2007;62:929-930.

doi: $10.1136 /$ thx.2007.082982

Correspondence to: Dr Alex G Little, Wright State University, Boonshoft School of Medicine,

Department of Surgery, 1 Wyoming Street, Suite 7801 WCHE, Dayton, Ohio 45409, USA; alex. little@wright.edu

Competing interests: None.

\section{REFERENCES}

1 Strand T-E, Rostad H, Dambuis RAM, et al. Risk factors for 30-day mortality after resection of lung cancer and prediction of their magnitude. Thorax 2007;62:991-7.

2 Little AG, Rusch WW, Bonner JA, et al. Patterns of surgical care of lung cancer patients. Ann Thorac Surg 2005;80:2051-6.

3 Silvestri GA, Handy J, Lackland D, et al. Specialists achieve better outcomes than generalists for lung cancer surgery. Chest 1998;114:675-80.

4 Goodney PP, Lucas FL, Stuckel TA, et al. Surgeon specialty and operative mortality with lung resection. Ann Surg 2005;241:179-84.

5 Bach PB, Cramer LD, Schrag D, et al. The influence of hospital volume on survival after resection for lung cancer. N Engl J Med 2001;345:181-8.

6 Paull DE, Thomas ML, Meade GE, et al. Determinants of quality of life in patients following pulmonary resection for lung cancer. Am J Surg 2006;192:565-71.

\section{Nicotine replacement therapy for smoking cessation in the "real world"}

\section{Saul Shiffman}

\section{NRT works in "real would" settings, but optimising its use would improve its impact on public health}

$\mathrm{T}$ he paper by West and Zhou published in this issue of Thorax (see page 998)

is an important contribution to the literature because it addresses and refutes the questions raised, on the basis of retrospective case-control studies, about the effectiveness of nicotine replacement therapy (NRT) in "real world" settings. ${ }^{1}$
Contrary to those retrospective analyses, West and Zhou's prospective population study found that NRT helps smokers to quit, roughly doubling the odds of successful quitting. The odds ratio of 2.2 reported by West and Zhou is quite consistent with the odds ratios reported in randomised clinical trials. ${ }^{3}$
In this sense, West and Zhou's finding that NRT is effective should come as no surprise. The efficacy of NRT has been demonstrated in over 100 randomised controlled trials encompassing over 35000 smokers. $^{3}$ Moreover, the effectiveness of NRT has also been demonstrated in multiple trials that tested NRT under overthe-counter conditions. ${ }^{4}$ These studies not only showed that over-the-counter NRT was more effective than placebo, but also that it was just as effective as using NRT under the care of a doctor. Although critics have argued that "effectiveness" (in contrast to "efficacy") has not been shown, in fact these trials were effectiveness trials: there was little or no screening or exclusion of participants, no extra support, instruction or therapy offered, and minimal data collection. ${ }^{5}$ The benefit of NRT has therefore been proven in both efficacy and effectiveness studies. West and Zhou's data extend this to a prospective study of 